\title{
ESTUDIO DE CARBONES ACTIVADOS IMPREGNADOS CON QUITOSANO Y SU COMPARACIÓN CON CARBONES COMERCIALES
}

\author{
Gerardo J. F. Cruz Cerro ${ }^{\mathrm{a}^{*}}$, Marlene Velásquez Yucra ${ }^{\mathrm{b}}$, Julio Santiago Contreras ${ }^{\mathrm{b}}$, \\ José L. Solís Veliz ${ }^{\mathrm{c}}$, Mónica M. Gómez León ${ }^{\mathrm{c}}$, Riitta Keiski ${ }^{\mathrm{d}}$
}

\section{RESUMEN}

Se obtuvieron carbones activados a partir de coronta de maíz (Zea mays) y pepa de ciruela (Spondia purpurae L.), los cuales fueron impregnados satisfactoriamente con quitosano. Las imágenes de microscopía electrónica de barrido de emisión de campo confirmaron la presencia de quitosano en cada uno de los carbones obtenidos.

Las propiedades adsorbentes y capacidad antimicrobiana frente a Escherichia coli fueron estudiadas y comparadas con las propiedades de dos carbones activados utilizados en filtros comerciales para la potabilización casera de agua. Los experimentos de equilibrio, empleando azul de metileno, mostraron que los carbones activados impregnados con quitosano poseen capacidades máximas de adsorción comparables con las de los carbones comerciales evaluados (entre 145 y $192 \mathrm{mg} / \mathrm{g}$ ).

Los carbones activados impregnados con quitosano presentaron una mejor respuesta microbiológica que la de los carbones comerciales. Tal es así que se obtuvo una mayor reducción de Escherichia coli. Los carbones activados preparados a partir de coronta de maíz alcanzó un nivel de reducción tan alto como 96,3\%. Mientras que para los carbones comerciales se tienen valores de reducción de menores a $60,3 \%$.

Palabras clave: Carbón activado, quitosano, propiedades adsorbentes, azul de metileno, Escherichia coli.

\section{ACTIVATED CARBONS IMPREGNATED BY CHITOSAN AND ITS COMPARISON WITH COMMERCIAL CARBONS}

\footnotetext{
ABSTRACT

Activated carbons were prepared from corncob and red mombin seed and impregnated with chitosan. Field Emission Scanning Microscopy (FESEM) micrographs confirm the

${ }^{a}$ Facultad de Ciencias Agrarias, Universidad Nacional de Tumbes, Av. Universitaria s/n Pampa Grande, Tumbes, Perú.gcruzc@untumbes.edu.pe

${ }^{\mathrm{b}}$ Facultad de Química e Ingeniería Química, Universidad Nacional Mayor de San Marcos, Av. Universitaria, Lima 1, Perú

${ }^{c}$ Facultad de Ciencias, Universidad Nacional de Ingeniería, Av. Túpac Amaru 210, Lima 25, Perú

${ }^{\mathrm{d}}$ Faculty of Technology, University of Oulu, P.O.Box 4300, FI-90014, Finland
} 
presence of chitosan in the activated carbon surface. Adsorptive and antibacterial properties of the prepared materials were compared with two adsorbents used in commercial water filters. Equilibrium adsorption experiments showed that the materials prepared in this study had maximum adsorption capacity to methylene blue that is comparable with those of the commercial adsorbents (between 145 and $192 \mathrm{mg} / \mathrm{g}$ ).

Activated carbons impregnated with chitosan exhibited higher antibacterial capacity against E. coli than the commercial adsorbents. The activated carbons prepared from corncob reach levels of reduction as higher as $96,3 \%$. Meanwhile the commercial activated carbons had reduction values lower than $60,3 \%$.

Key words: Agrowastes, activated carbon, chitosan, methylene blue, adsorption properties, Escherichia coli.

\section{INTRODUCCIÓN}

Actualmente, existe en el mercado una serie de filtros utilizados para asegurar la calidad del agua potable a nivel casero. La mayoría de ellos están hechos a base de carbón activado en distintas presentaciones (granular, entrelazado con fibras de celulosa y en forma de monolitos) los cuales a su vez están impregnados con nanopartículas de diferentes componentes, entre los que destaca la plata (Ag). Sin embargo, la totalidad de estos productos son importados y de alto costo, por lo cual son difíciles de ser adquiridos masivamente.

Si bien es cierto la tecnología de nanopartículas de Ag ha sido utilizada exitosamente como antimicrobiano en el tratamiento de aguas ${ }^{1}$, la Ag no deja de ser un material tóxico para los ecosistemas acuáticos ${ }^{2}$, afecta la flora benéfica del suelo ${ }^{3}$, sus iones libres es potencialmente tóxico para el ser humano ${ }^{4}$, y es una materia prima no renovable. Además, para la producción del carbón activado se utiliza carbón mineral, el cual es un material no renovable que podría ser utilizado más eficientemente en la producción de energía en lugar de materia prima para la producción de materiales adsorbentes para el tratamiento de agua.

En esta coyuntura se hace necesaria la búsqueda de materias primas renovables y de bajo costo que reemplacen a las tradicionales utilizadas para producir estos materiales para el tratamiento de agua. Una de las alternativas es el uso de residuos agrícolas, como precursores de carbones activados. Se ha comprobado que estos materiales lignocelulósicos son precursores idóneos para la producción de carbones activados con alta porosidad, y sobre todo alta mesoporosidad, además han sido usados exitosamente en la adsorción de contaminantes de aguas ${ }^{5,6}$. Sin embargo, no poseen una de las principales propiedades que deben tener los materiales utilizados en los filtros de agua, es decir no poseen capacidad antibacteriana. Por ello, en el presente estudio se planteó producir un material compósito: carbón activado-quitosano que podría ser usado en filtros comerciales. Se sabe que el quitosano tiene propiedades antimicrobiales muy interesantes y que además es biocompatible ${ }^{7}$. Adicionalmente, el quitosano se puede obtener de residuos hidrobiológicos, lo que coloca los resultados del 
presente estudio en una opción tecnológica-ambiental interesante. En el presente trabajo el carbón activado se obtuvo de coronta de maíz (Zea mays) y pepa de ciruela (Spondia purpurae L.), los cuales fueron impregnados con quitosano comercial marca Fluka. Los estudios comparativos con los productos comerciales se llevaron a cabo morfológicamente mediante estudios de microscopía electrónica de barrido de emisión de campo, estudios de equilibrio y evaluación de la eficiencia de la adsorción del azul de metileno. Finalmente, se evaluó la capacidad de reducción de Escherichia coli.

\section{PARTE EXPERIMENTAL}

Dos muestras de carbones activados fueron preparadas a partir de coronta de maíz (Zea mays) y de pepa de ciruela (Spondia purpurae L.). La activación realizada fue de tipo química utilizando $\mathrm{ZnCl}_{2}$ en una proporción $1 / 1$ con la materia prima, la cual se realizó paralelamente con un proceso de carbonización a $600{ }^{\circ} \mathrm{C}$ durante $2 \mathrm{~h}$ en atmósfera de nitrógeno. El carbón obtenido se lavó con una solución de ácido clorhídrico $0,5 \mathrm{~N}$ y abundante agua destilada para luego ser secado por $12 \mathrm{~h} \mathrm{a} 100{ }^{\circ} \mathrm{C}^{8}$. El quitosano se soportó en los carbones mediante impregnación clásica. $1 \mathrm{~g}$ de quitosano se mezcló en una solución de ácido acético al $1 \%$, se colocó en un reactor y se elevó la temperatura hasta $100^{\circ} \mathrm{C}$ y a una presión de 20 psi durante un periodo de $1 \mathrm{~h}$. Los materiales obtenidos se lavaron con abundante agua destilada y se secaron a $40{ }^{\circ} \mathrm{C}$ hasta peso constante.

Los materiales obtenidos se identificaron como CM-Q y CP-Q para los materiales obtenidos de la coronta de maíz y la pepa de ciruela, respectivamente.

Adicionalmente, dos materiales utilizados en filtros comerciales fueron extraídos, molidos y tamizados a tamaño de partícula menor de $0,25 \mathrm{~mm}$. Los carbones comerciales se identificaron como CC1 y CC2.

Los estudios de morfología por microscopía electrónica de barrido de emisión de campo se realizaron en carbones obtenidos de residuos agrícolas sin impregnar e impregnados con quitosano, así como con carbones comerciales. Para estos estudios se empleó un microscopio electrónico de barrido de emisión de campo, marca Zeiss, modelo Ultra plus. Se realizaron estudios estructurales de cristalinidad de los carbones comerciales mediante difracción de rayos X, para lo cual se usó un difractómetro Siemens D5000, con una radiación CuK $\alpha$.

Para comparar las capacidades de adsorción de los carbones obtenidos con los productos de los filtros comerciales, se efectuaron pruebas de equilibrio y cinética con azul de metileno. Es de precisar que el azul de metileno es utilizado comúnmente como modelo comparativo para evaluar la capacidad de adsorción de carbones activados ${ }^{8}$ por su facilidad de medición y la posibilidad de tener amplia información de la cinética de adsorción de diversos carbones. Las pruebas de equilibrio de adsorción se llevaron a cabo utilizando 5 soluciones de 100 $\mathrm{ml}$ de azul de metileno con concentraciones en el rango de 40 y $120 \mathrm{mg} / \mathrm{l}$. Cada solución se colocó en matraces de $250 \mathrm{ml}$ y se adicionó en cada uno de ellos $0,1 \mathrm{~g}$ de carbón. Los 
matraces fueron agitados a $180 \mathrm{rpm}$ en un agitador orbital durante $24 \mathrm{~h}$ a una temperatura de $30 \pm 2{ }^{\circ} \mathrm{C}$. Luego de las $24 \mathrm{~h}$, se extrajo una alícuota de la solución, se filtró y se midió la absorbancia a una longitud de onda de $660 \mathrm{~nm}$ de la solución en un espectrofotómetro UVVIS Spectroquant Pharo 300 (Merck), luego con una curva de calibración se determinó la concentración del azul de metileno en la solución. El mismo procedimiento se repitió para cada muestra.

Para ajustar los datos de equilibrio se utilizó el modelo de Langmuir:

$$
\frac{C_{e}}{q_{e}}=\frac{1}{K_{L} q_{\max }}+\frac{C_{e}}{q_{\max }}
$$

donde $q_{e}$ qe es la cantidad de azul de metileno adsorbido en el equilibrio ( $\left.\mathrm{mg} / \mathrm{g}\right), C_{e}$ es la concentración de equilibrio del azul de metileno adsorbido (mg/l), $q_{\max } \mathrm{y} K L$ son las constantes del modelo relativas a la máxima capacidad de adsorción (capacidad en la monocapa) y la energía de adsorción, respectivamente.

Para las pruebas de cinética se utilizó una solución de $10 \mathrm{mg} / \mathrm{l}$ de azul de metileno y una carga inicial de carbón activado de $0,1 \mathrm{~g} / \mathrm{l}$. Esta solución de $150 \mathrm{ml}$ se colocó en un matraz de $250 \mathrm{ml}$ y se agitó durante todo el experimento. Para monitorear la concentración del azul de metileno se extrajeron alícuotas (hasta un tiempo de $240 \mathrm{~min}$ ) que luego fueron filtradas y posteriormente medidas espectrofotométricamente de manera análoga que para las pruebas de equilibrio. Para ajustar los datos de cinética se utilizó el modelo de pseudo-segundo orden:

$$
\frac{t}{q_{t}}=\frac{1}{K_{2} q_{e}^{2}}+\frac{t}{q_{e}}
$$

donde $t$ es el tiempo de adsorción ( $\min$ ), $q_{t}$ es la cantidad de azul de metileno adsorbida en el tiempo $\mathrm{t}(\mathrm{mg} / \mathrm{g}), \mathrm{k}_{2}$ es la constante de pseudo-segundo orden $(\mathrm{g} / \mathrm{mg} \cdot \mathrm{min})$ y $q_{e}$ es cálculo de la capacidad de adsorción en el equilibrio $(\mathrm{mg} / \mathrm{g})$. Para obtener los parámetros del modelo se graficó t/qt en función del tiempo.

Los estudios de capacidad antibacteriana se realizaron frente a la cepa bertani Escherichia coli (ATCC 25922), las cuales fueron sembradas en caldo Luria a $36{ }^{\circ} \mathrm{C}+/-1$ durante $24 \mathrm{~h}$. Una alícuota del cultivo fue suspendida en $10 \mathrm{ml}$ de solución salina fisiológica hasta obtener una suspensión microbiana equivalente al tubo número 0,5 del nefelómetro de McFarland. Se tomó de la suspensión $0,1 \mathrm{ml}$ para los tiempos de contacto de 30 y $60 \mathrm{~min}$. Estas muestras fueron diluidas y luego sembradas en agar Muller-Hinton e incubadas a $37^{\circ} \mathrm{C}$ por $24 \mathrm{~h}{ }^{9-10}$. Se hizo un recuento de las colonias y se calculó la capacidad de reducción en \% respecto de la cantidad de microorganismo inicial.

\section{RESULTADOS Y DISCUSIÓN}

La figura 1 muestra las micrografías de microscopía electrónica con diferentes magnificaciones de los carbones activados obtenidos de coronta de maíz (a) y pepa de ciruela (b), así como 
carbones activados obtenidos de coronta de maíz con impregnación de quitosano, CM-Q (c y e) y de pepa de ciruela con impregnación de quitosano, CP-Q (d y f).

La micrografía correspondiente al carbón activado procedente de la coronta de maíz presenta una estructura muy porosa, constituida por cavidades o poros de tamaños bastante uniformes que se encuentran en el rango de 10 y $20 \mu \mathrm{m}$ (figura 1a), mientras que para la micrografía correspondiente al carbón activado procedente de la pepa de ciruela se aprecian también poros, con una dispersión mayor de tamaños que se encuentran entre 10 y $30 \mu \mathrm{m}$ (figura 1b). Ambas muestras presentan algunas estructuras sólidas que no forman parte de las paredes del carbón activado, las cuales presentan un tamaño en el orden de micrómetros. Estas estructuras podrían estar relacionadas con el agente químico activador, o con pequeños trozos de los mismos carbones que se producen durante la molienda de las muestras.

La muestra CM-Q, presenta una morfología similar al del carbón activado sin impregnación procedente de la misma materia prima (Fig. 1a), se observan cavidades de tamaños muy uniformes y además orificios en las paredes de las cavidades (Fig. 1c). En la micrografía a mayor magnificación (Fig. 1e) se observa claramente que las paredes del carbón activado muestran en su superficie unas estructuras ovoidales que en su contorno presentan mayor concentración de material. Estas estructuras están en el orden de 500 a 700 nm y se atribuyen al quitosano.

La figura 1d muestra la micrografía de la muestra PC-Q, se observa claramente la presencia de cavidades, que fueron identificadas para el caso del carbón activado sin impregnación (Fig. 1b). Pero para una mayor magnificación (Fig. 1f) se puede observar claramente la presencia de estructuras granulares en la superficie de los carbones, las cuales son asignadas al quitosano impregnado. 


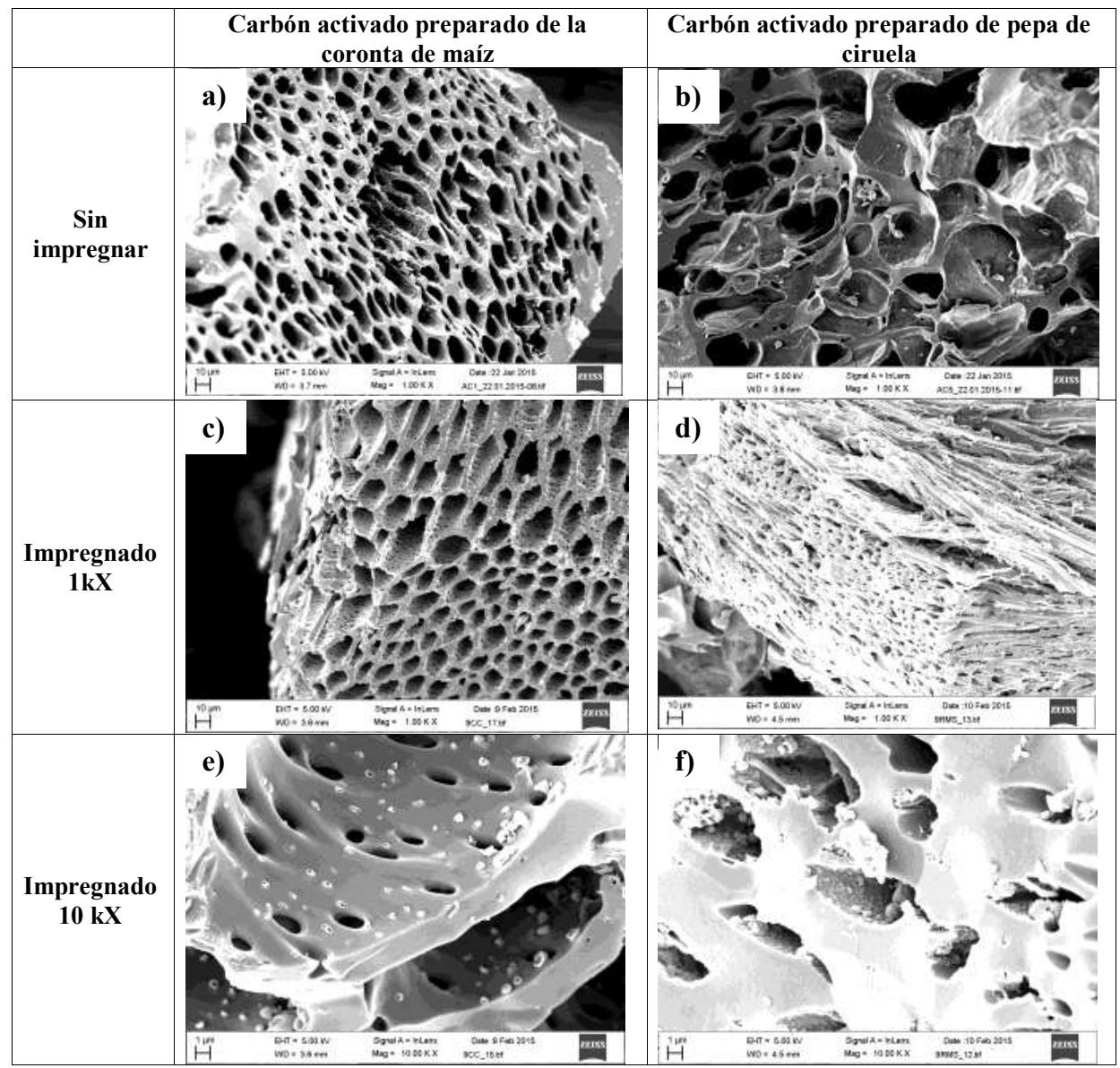

Figura 1. Micrografías electrónicas de carbones activados producidos de residuos agroindustriales: (a) coronta de maíz y (b) pepa de ciruela, así como los carbones impregnados con quitosano, obtenidos de coronta de maíz, CM-Q (c y e) y de pepa de ciruela, PC-Q (d y f).

En la figura 2 se muestran fotografías y micrografías de microscopía electrónica de las dos muestras de carbon activado extraídas de filtros comerciales utilizados comúnmente en el tratamiento casero de agua potable $\mathrm{CC} 1$ y CC2. El material $\mathrm{CC} 1$ es un carbón activado granular (Fig. 2a), mientras que el material CC2 es un cárbon activado entrelazado con fibras de celulosa (cloth activated carbon), el cual se encuentra compactado y asegurado con una malla de plástico, tal como se muestra en la figura $2 b$.

Las micrografías de miscroscopía electrónica (figuras 2 c y d) muestran la morfología de ambos carbones activados. Los granulos del carbon activado CC1 son de forma tetrahédrica y aparentemente altamente porosos, con poros de varias formas desde hexagonales hasta irregulares (Fig. 2c). El carbon activado CC2 (Fig. 2d) presenta granos de diversos tamaños y formas, mostrándose el entrelazamiento con las fibras de celulosa ya mencionado. 


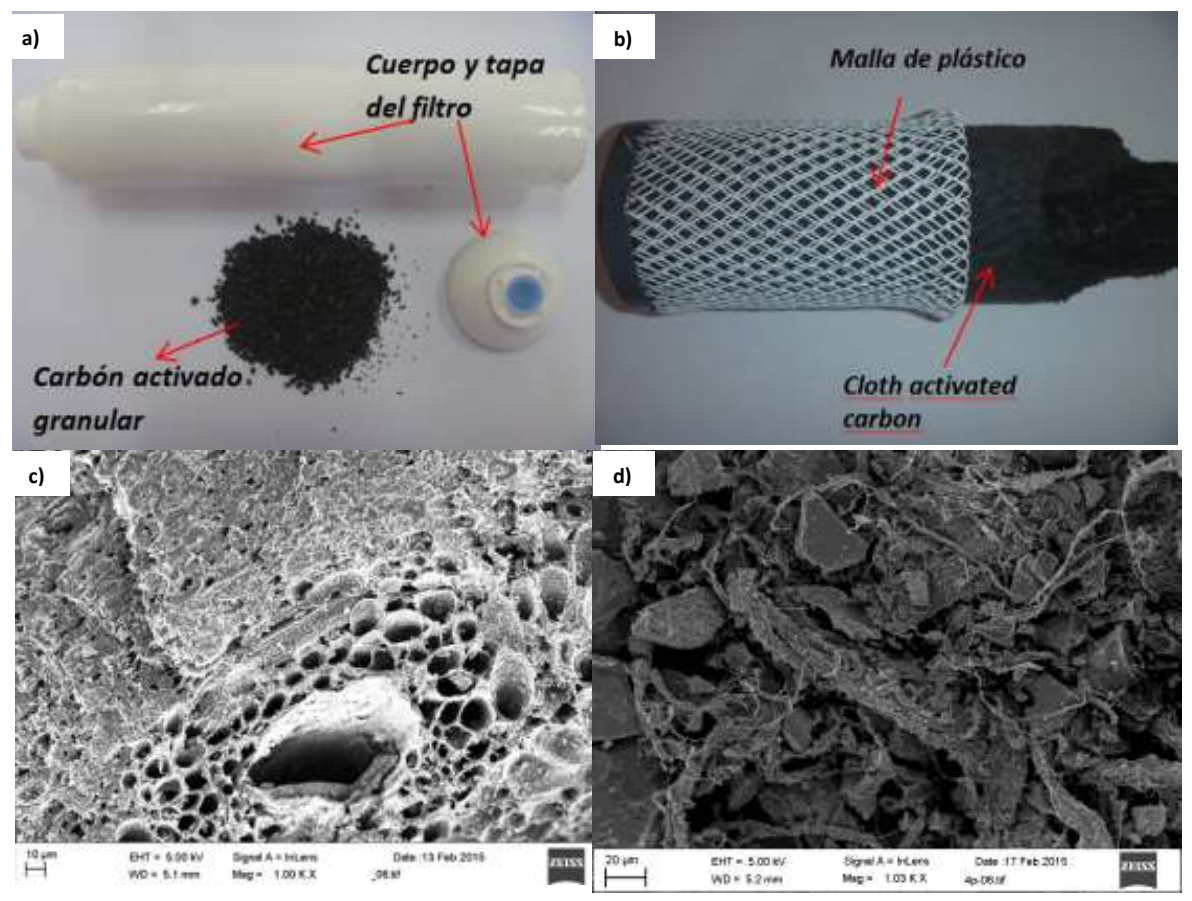

Figura 2. Fotografías de los filtros comerciales seleccionados para el estudio (a y b). Imágenes de microscopia electrónica de los materiales comerciales CC1(c) y CC2 (d).

Los difractogramas de rayos X de las muestras comerciales (Fig. 3) corroboran lo encontrado por el análisis EDS de la superficie, encontrando plata en la muestra CC1 y no en la CC2. Además, ambas muestras presentan los picos anchos clásicos de los carbones activados alrededor de $2 \theta=24$ y $2 \theta=42$ (11).

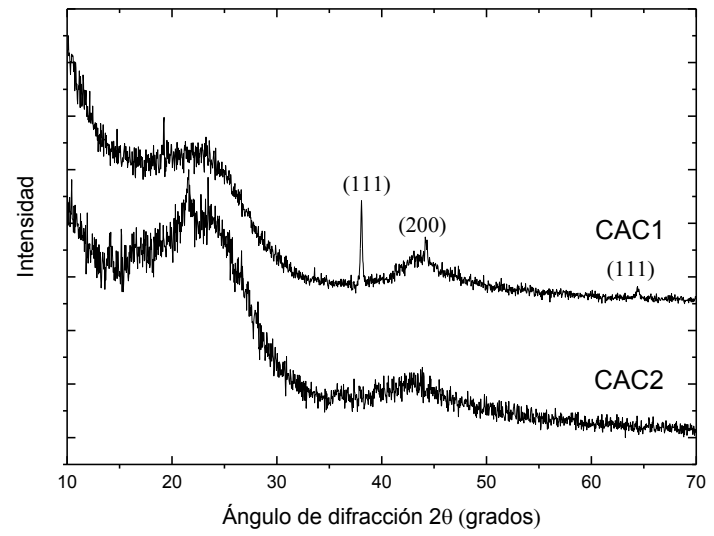

Figura 3. Difractogramas de rayos $\mathrm{X}$ para los carbones activados comerciales, $\mathrm{CAC} 1$ y CAC2. Los picos señalados en CAC1 corresponden a los índices de Miller de las reflexiones de los planos cristalográficos correspondientes a la plata. 
La figura 4 contiene los datos de equilibrio de las dos muestras de carbones impregnadas con quitosano y de las muestras comerciales. En todos los casos a mayor concentración inicial $\left(C_{0}\right)$, la cantidad de azul de metileno adsorbida en el equilibrio $\left(q_{e}\right)$ es mayor; lo que está en concordancia con lo mencionado por Cherifi et al. (12) quien plantea que este hecho está basado en que la concentración inicial de azul de metileno provee una importante fuerza motriz para sobrepasar la resistencia por la transferencia de masa, por lo que a mayor concentración inicial mayor será la cantidad adsorbida al sobrepasar más fácil y rápidamente esta resistencia.

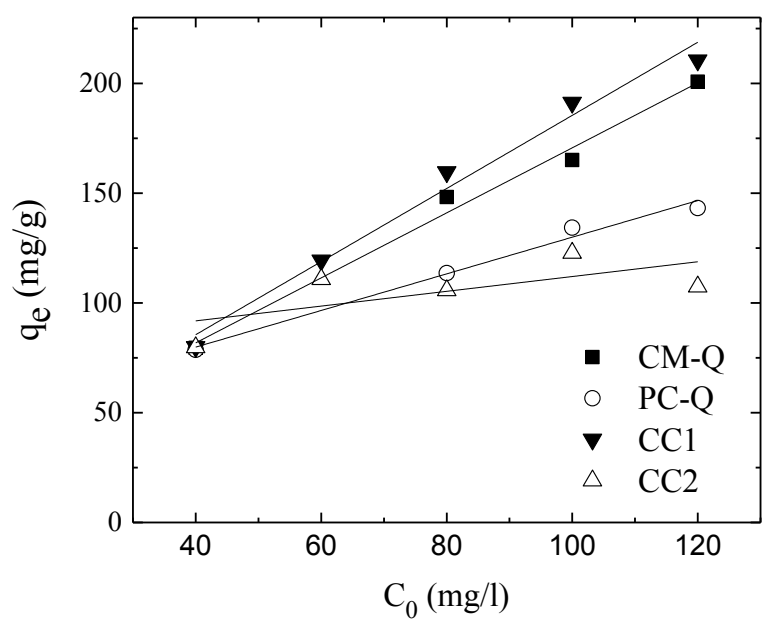

Figura 4. Datos de equilibrio de adsorción de azul de metileno por los materiales preparados y los carbones comerciales - evolución de la concentración de equilibrio en función de la concentración inicial.

Todos los datos de equilibrio se ajustan al modelo de Langmuir, tal como se aprecia al analizar los respectivos valores de $R^{2}$ mostrados en la tabla 1 , que varían entre 0,97 y 0,99 . Esto supone una adsorción homogénea del azul de metileno sobre la monocapa de los materiales analizados.

Comparando las máximas capacidades de adsorción $\left(q_{\max }\right)$ de los materiales producidos y la de los carbones activados comerciales (tabla 1), el carbón CC2 muestra la mayor capacidad de adsorción de azul de metileno, seguido del carbón CM-Q, el PC-Q y, finalmente, del otro carbón comercial CC1; por lo que se demuestra que los carbones activados impregnados con quitosano poseen una capacidad de adsorción de azul de metileno comparable a los carbones comerciales estudiados. 
Tabla 1. Parámetros obtenidos de la aplicación del modelo de equilibrio de Langmuir de los datos de adsorción de azul de metileno por los materiales preparados y los materiales comerciales.

\begin{tabular}{ccccc}
\hline \multirow{2}{*}{ Parámetro } & \multicolumn{4}{c}{ Muestras } \\
\cline { 2 - 5 } & CM-Q & PC-Q & CC1 & CC2 \\
\hline$q_{\max }(m g / g)$ & 192 & 145 & 110 & 213 \\
$K_{L}(l / g)$ & 0,98 & 0,38 & 2,46 & 7,83 \\
$R^{2}$ & 0,97 & 0,98 & 0,99 & 0,99 \\
\hline
\end{tabular}

Las cantidades adsorbidas de azul de metileno en función del tiempo (qt) son mayores para el caso de los carbones comerciales entre los primeros 100 min (Fig. 5 a). Sin embargo, después del tiempo mencionado, los valores de qt son tan iguales o incluso mayores para las muestras CM-Q y PC-Q.

Comparando las dos muestras producidas en el presente estudio, se aprecia que la muestra PC-Q presenta ligeramente mayores valores de qt que la muestra CM-Q en todos los tiempos analizados.
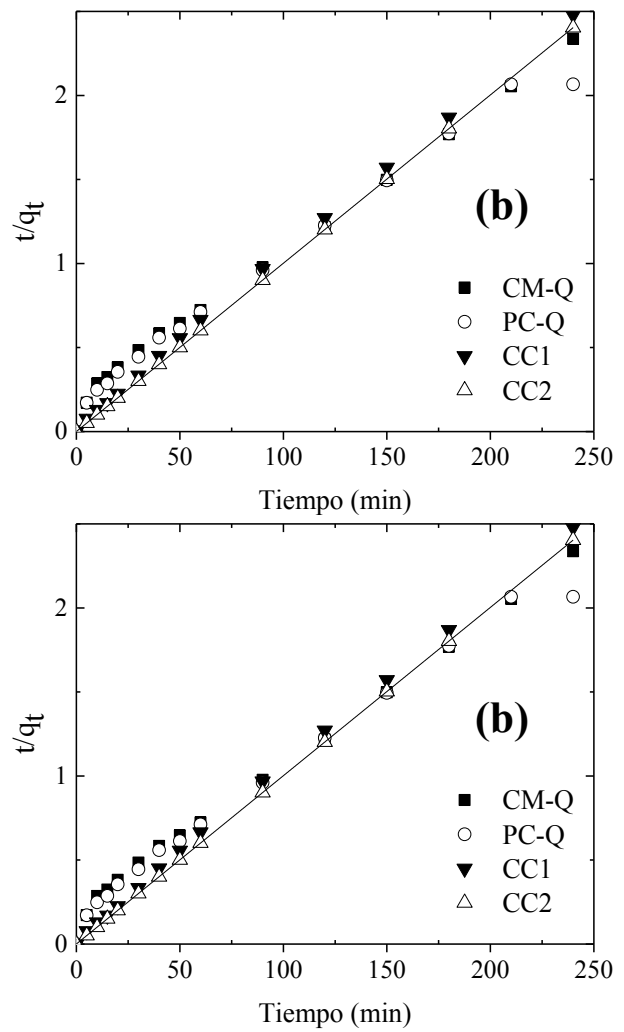

Figura 5. (a) Datos de cinética de adsorción de azul de metileno para los carbones impregnados con quitosano (CM-Q y PC-Q) y comerciales (CC1 y CC2); (b) Ajuste de los datos de equilibrio al modelo de pseudo-segundo orden. 
Los datos de cinética de adsorción de azul de metileno se ajustan al modelo de pseudosegundo orden, tal como lo demuestran los valores de $R^{2}$ obtenidos del modelo y estén cercanos a la unidad, entre 0,989 y 1 (tabla 2). Este hecho supone una interacción del tipo químico entre el azul de metileno y los grupos funcionales superficiales de los materiales adsorbentes. Los valores de las cantidades de azul de metileno adsorbidas en el equilibrio $\left(q_{e}\right)$ son mayores para el caso de los materiales producidos en el presente estudio, corroborando la tendencia encontrada anteriormente cuando se analizó $q_{t}$ en función del tiempo.

Tabla 2. Parámetros de cinética de pseudo-segundo orden de la adsorción de azul de metileno por las muestras producidas y los carbones comerciales.

\begin{tabular}{ccccc}
\hline \multirow{2}{*}{ Parámetro } & \multicolumn{4}{c}{ Muestra } \\
\cline { 2 - 5 } & CM-Q & PC-Q & CC1 & CC2 \\
\hline$q_{e}(\mathrm{mg} / \mathrm{g})$ & 111 & 115 & 97 & 100 \\
$k_{2}(\mathrm{~g} / \mathrm{mg} \cdot \mathrm{min})$ & 0,0004 & 0,0004 & 0,0038 & 0,004 \\
$R^{2}$ & 0,995 & 0,989 & 0,9997 & 1 \\
\hline
\end{tabular}

Comparando los valores de $k_{2}$ (tabla 2) se puede evidenciar que la muestra CC2 posee una mayor velocidad de reacción que el resto de muestras. CM-Q, PC-Q y CC1 presentan velocidades de reacción similares. Esto podría sostenerse en la posibilidad de una mayor cantidad de poros presentes en la muestra $\mathrm{CC} 2$, de tal manera que permiten la adsorción de la molécula de azul de metileno que tiene un tamaño de $1,66 \times 0,82 \times 0,54 \mathrm{~nm}^{8}$. Otro de los factores es el tamaño de partícula del material; tal como se aprecia en las micrografías de microscopia electrónica (figura 2) a pesar de que la muestra CC2 también fue tamizada a un tamaño de partícula menor de $0,25 \mathrm{~mm}$, esta muestra un tamaño de partícula reducido comparado con el tamaño de partícula del resto de muestras. A menor tamaño de partícula mas fácil y rápido acceso del adsorbato a la superficie del material donde se encuentran los sitios activos de adsorción y además existen más sitios activos disponibles ${ }^{13}$.

Tabla 3. Reducción (\%) de E. coli por los materiales producidos Comparativo con carbones activado comerciales

\begin{tabular}{ccccc}
\hline Tiempo de & \multicolumn{4}{c}{ Muestra } \\
\cline { 2 - 5 } contacto & CM-Q & PC-Q & CC1 & CC2 \\
\hline 30 min & 86,1 & 59,3 & 43,3 & 40,5 \\
60 min & 85,1 & 96,3 & 48,9 & 60,3 \\
\hline
\end{tabular}

En general, los materiales producidos en el presente estudio presentan mejor capacidad de reducción de $E$. coli que los carbones comerciales, llegando a valores de eliminación de entre 85,1 y $96,3 \%$ después de 60 min de tiempo de contacto para las muestras CM-Q y PC-Q, respectivamente (tabla 3). De acuerdo a los resultados de 30 y $60 \mathrm{~min}$ de tiempo de contacto, la muestra CM-Q llega a su máxima capacidad de reducción de $\boldsymbol{E}$. coli en los primeros 30 min, mientras que la muestra PC-Q alcanza su máxima capacidad de reducción mucho después (después del tiempo analizado), sin embargo su capacidad máxima de reducción de E. coli es mayor que la de la muestra CM-Q. Los carbones activados comerciales CC1 y CC2 mostraron niveles de reducción de E. coli de 48,9 y 60,3\% luego de 60 min de tiempo de contacto, respectivamente. 
La capacidad antibacteriana del quitosano contra las bacterias Gram negativas como la E. coli ha sido estudiada por varios autores ${ }^{14,15}$ y coincide con lo encontrado en el presente estudio.

Acorde a los resultados de los carbones activados comerciales, el carbón con alto contenido de cloro presentó mayor capacidad de eliminación de $E$. coli que el que contiene nanopartículas de plata.

\section{CONCLUSIONES}

Se han producido satisfactoriamente carbones activados a partir de coronta de maíz (Zea mays) y pepa de ciruela (Spondia purpurae L.) impregnados con quitosano (CM-Q y PCQ). Imágenes de microscopia electrónica confirman la presencia de quitosano en diferentes morfologías en cada uno de los carbones activados producidos. En la muestra CM-Q el quitosano se encuentra como partículas individuales, mientras que en la muestra PC-Q como partículas individuales y también aglomeraciones.

Los experimentos de equilibrio mostraron que los carbones activados impregnados con quitosano mostraron comparables capacidades máximas de adsorción de azul de metileno que con dos carbones activados utilizados en filtros comerciales de agua (entre 145 y 192 $\mathrm{mg} / \mathrm{g}$ ) de acuerdo al modelo de Langmuir.

Los datos cinéticos de los adsorbentes obtenidos en el presente estudio y de los comerciales se ajustaron al modelo de pseudo-segundo orden, suponiendo una interacción de tipo químico entre el azul de metileno y las muestras. Estas pruebas mostraron mayores cantidades adsorbidas de azul de metileno en la primera etapa de los experimentos para los carbones comerciales, sin embargo los carbones producidos en el presente estudio resultaron con mayores capacidades de adsorción en el equilibrio.

Los carbones activados impregnados con quitosano presentaron mayor nivel de reducción de E. coli que los carbones comerciales, llegando a valores de reducción de entre 85,1 y 96,3\% después de 60 min de tiempo de contacto para las muestras CM-Q y PC-Q, respectivamente.

\section{AGRADECIMIENTOS}

Los investigadores agradecen el apoyo económico de la Universidad Nacional de Tumbes (Proyecto Canon Resolución $N^{\circ}$ 0722-2014/UNT-R), al Programa Nacional de innovación para la competitividad y productividad-INNOVATE Perú (Contrato $\mathrm{N}^{\circ}$ 356-PNICPPIAP-2014). A la Academia de Finlandia también se le agradece por el financiamiento a través del proyecto AdMatU (DNo: 269631). M.M.G agradece al Instituto General de Investigación de la Universidad Nacional de Ingeniería (IGI-UNI). 


\section{REFERENCIAS BIBLIOGRÁFICAS}

1. Rai M, Yadav A, Gade A. Silver nanoparticles as a new generation of antimicrobials. Biotechnology advances. 2009; 27(1):76-83.

2. Kwak J I, Cui R, Nam S H, Kim S W, Chae Y, An Y J. Multispecies toxicity test for silver nanoparticles to derive hazardous concentration based on species sensitivity distribution for the protection of aquatic ecosystems. Nanotoxicology. 2016; 10(5), 521-530.

3. Murata T, Kanao-Koshikawa M, Takamatsu T. Effects of $\mathrm{Pb}, \mathrm{Cu}, \mathrm{Sb}, \mathrm{In}$ and $\mathrm{Ag}$ contamination on the proliferation of soil bacterial colonies, soil dehydrogenase activity, and phospholipid fatty acid profiles of soil microbial communities. Water Air Soil Pollution, 2005;164 :103-118.

4. Wijnhoven S W, et. al. Nano-silver-a review of available data and knowledge gaps in human and environmental risk assessment. Nanotoxicology. 2009; 3(2), 109-138.

5. Leelavathy K R, Nageshwaran V, Bharathi M. Comparative Study on Commercial and Corn Cobs Activated Carbon for Removal of Congo Red Dye. Applied Mechanics and Materials. 2015;787, 233-237.

6. Mor S, Chhoden K, Ravindra K. Application of agro-waste rice husk ash for the removal of phosphate from the wastewater. Journal of Cleaner Production. 2016;129, 673-680.

7. Krajewska B.. Application of chitin-and chitosan-based materials for enzyme immobilizations: a review. Enzyme microb. Technol., 2004;35(2):126-139.

8. Cruz G. et.al., A Comparative Study on Activated Carbons Derived from a Broad Range of Agro-industrial Wastes in Removal of Large-Molecular-Size Organic Pollutants in Aqueous Phase. Water, Air, \& Soil Pollution. 2015;226(7):1-15.

9. Ortiz-Ibarra H. et. al.. Surface characterization of electroposited silver on activated carbon for bactericidal purposes. Journal of colloid and interface science. 2007;314(2):562-571

10. Srinivasan NR, Shankar PA, Bandyopadhyaya R. Plasma treated acBtivated carbon impregnated with silver nanoparticles for improved antibacterial effect in water desinfection. Carbon. 2013;57:1-10.

11. Acharya J, Sahu JN, Mohanty CR, Meikap BC. Removal of lead (II) from wastewater by activated carbon developed from Tamarind wood by zinc chloride activation. Chemical Engineering Journal. 2009;149(1):249-262.

12. Cherifi H, Fatiha B, Salah H. Kinetic studies on the adsorption of methylene blue onto vegetal fiber activated carbons. Applied Surface Science. 2013;282:52-59.

13. El Qada EN, Allen SJ, Walker GM. Adsorption of methylene blue onto activated carbon produced from steam activated bituminous coal: a study of equilibrium adsorption isotherm. Chemical Engineering Journal. 2006;124(1):103-110.

14. Fernandez-Saiz P, Lagaron JM, Ocio MJ. Optimization of the biocide properties of chitosan for its application in the design of active films of interest in the food area. Food Hydrocolloids. 2009;23:913-921.

15. Kong M, Guang Chen X, Xing K, Jin Park H. Antimicrobial properties of chitosan and mode of action: A state of the art review. International Journal of Food Microbiology. $2010 ; 144: 51-63$ 